\title{
Endoscopic endonasal extreme far-medial approach for a lower clivus osteochondroma in a patient with hereditary multiple exostoses: illustrative case
}

\author{
Yusuke Morinaga, MD, ${ }^{1}$ Hiroyoshi Akutsu, MD, PhD, ${ }^{1}$ Hiroyoshi Kino, MD, PhD, ${ }^{1}$ Shuho Tanaka, MD, PhD, ${ }^{2}$ Hidetaka Miyamoto, $\mathrm{MD},{ }^{2}$ \\ Masahide Matsuda, MD, PhD, ${ }^{1}$ Muneyoshi Yasuda, MD, PhD, ${ }^{3}$ and Eiichi Ishikawa, MD, $\mathrm{PhD}^{1}$
}

Departments of ${ }^{1}$ Neurosurgery and ${ }^{2}$ Otolaryngology, Faculty of Medicine, University of Tsukuba, Tsukuba, Ibaraki, Japan; and ${ }^{3}$ Department of Neurosurgery, Ichinomiyanishi Hospital, Ichinomiya, Aichi, Japan

\begin{abstract}
BACKGROUND The authors reported on the use of endoscopic endonasal surgery (EES) for clivus osteochondroma in a patient with hereditary multiple exostoses (HME), a rare pediatric disorder characterized by the formation of osteochondromas adjacent to the growth plates of the axial and appendicular skeletal elements.

OBSERVATIONS A 26-year-old man with a family history of HME reported progressive hoarseness and dysphagia over the previous 6 months. He was referred to us after magnetic resonance imaging (MRI) showed a bone tumor in the lower clivus. MRI revealed tumor proliferation in the lower clivus and its extension to the bilateral occipital condyle and jugular tubercle. The hypoglossal canal and jugular foramen were encased on the right side, whereas the medulla oblongata was compressed. The tumor was subtotally resected with EES, and the brainstem was successfully decompressed. The pathological diagnosis was exostoses. Transient postoperative worsening of dysphagia improved within 1 month without other neurological deficits. The patient underwent posterior occipitoaxial fixation 3 months after EES to correct instability and local lateral tilt of the right atlanto-occipital joint.
\end{abstract}

LESSONS The authors' experience showed that EES is effective for resection of lower clivus osteochondromas, including the cartilaginous cap, and may improve clinical outcomes in patients with HME.

https://thejns.org/doi/abs/10.3171/CASE2153

KEYWORDS cartilaginous cap; clivus; endoscopic endonasal surgery; hereditary multiple exostoses

Hereditary multiple exostoses $(\mathrm{HME})^{1-6}$ is a rare pediatric disorder caused by loss-of-function mutations in the genes that encode the heparan sulfate-synthesizing enzymes EXT1, EXT2, and EXT3. ${ }^{7}$ HME is characterized by the formation of cartilaginous outgrowths, called "osteochondromas," next to the growth plates of axial and appendicular skeletal elements. Given the lack of surgical reports concerning skull base lesions, it is unknown whether these tumors also develop into endochondral elements of the craniofacial skeleton. ${ }^{1-6}$ To the best of our knowledge, this is the first study to document the use of endoscopic endonasal surgery (EES) to treat a clivus lesion in a patient with HME. Herein, we describe the surgical procedure and the patient's clinical course.

\section{Illustrative Case}

\section{History and Presentation}

A 26-year-old man with a family history of HME had undergone multiple operations to address HME lesions in the right forearm, thoracic vertebra, and bilateral lower legs during childhood. He consulted a nearby clinic and reported mainly hoarseness and dysphagia over the previous 6 months, but magnetic resonance imaging (MRI) showed a bone tumor in the lower clivus, and he was referred to our hospital. Computed tomography (CT) (Fig. 1) revealed a bone tumor that had proliferated into the lower clivus and extended to the jugular tubercle and occipital condyle. T1- and T2-weighted MRI (Fig. 2) showed a heterogeneous high-signal lesion in the lower

\footnotetext{
ABBREVIATIONS BMP = bone morphogenetic protein; $\mathrm{CN}=$ cranial nerve; CSF = cerebrospinal fluid; CT = computed tomography; EES = endoscopic endonasal surgery; HGC = hypoglossal canal; HME = hereditary multiple exostoses; ICA = internal carotid artery; JF = jugular foramen; MRI = magnetic resonance imaging; $\mathrm{NIM}=$ nerve integrity monitor.
}

INCLUDE WHEN CITING Published May 17, 2021; DOI: 10.3171/CASE2153.

SUBMITTED January 28, 2021. ACCEPTED February 19, 2021.

(C) 2021 The authors, CC BY-NC-ND 4.0 (http://creativecommons.org/licenses/by-nc-nd/4.0/) 

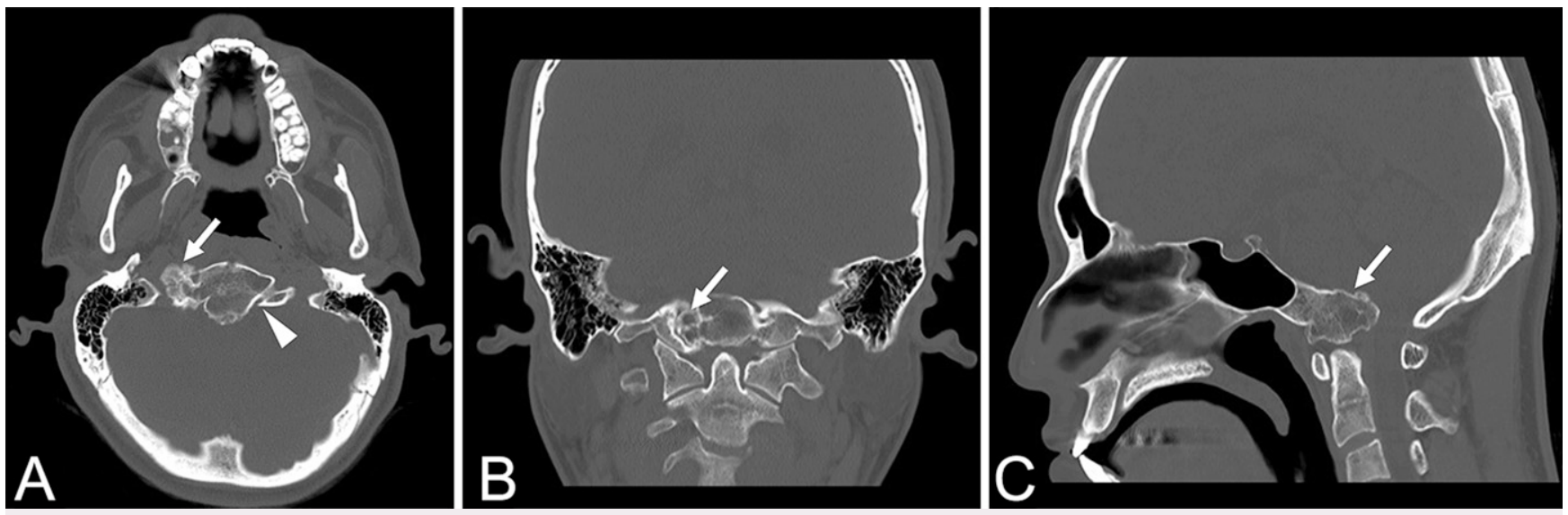

FIG. 1. Preoperative CT. A: Axial CT view showing proliferation of the osteoblastic tumor encasing the right HGC (arrow). Meanwhile, the left HGC remains intact, and the cortex of the canal is preserved (arrowhead). B: Coronal CT view showing rightward dominance of the tumor progression toward the occipital condyle (arrow). C: Sagittal CT view showing extension of the tumor in the lower clivus, protruding posteriorly at the craniocervical junction (arrow).
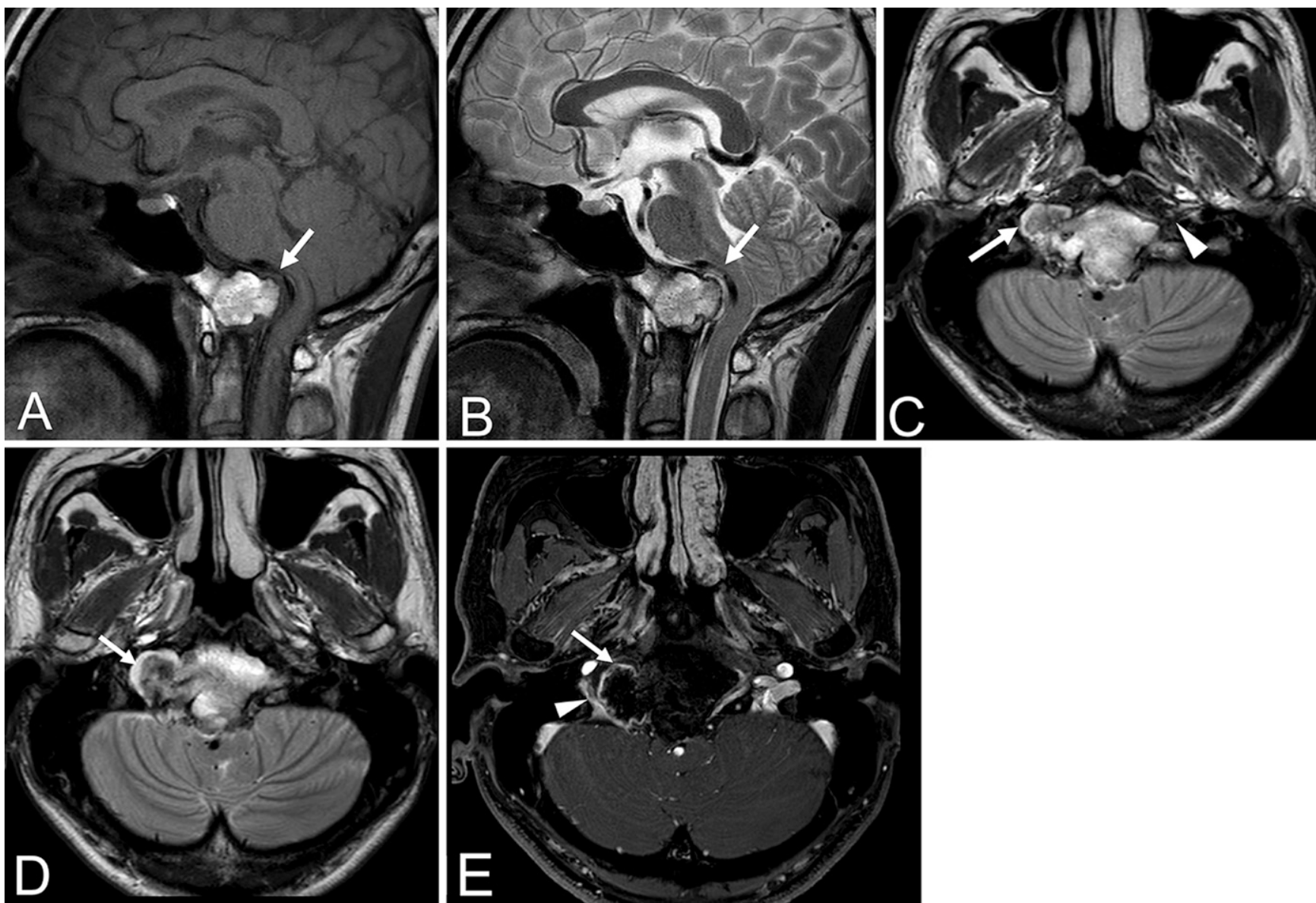

FIG. 2. Preoperative MRI. Sagittal views of the T1- (A) and T2- (B) weighted images revealing a heterogeneous high-signal lesion in the lower clivus (arrows). The medulla oblongata was compressed by the tumor. C: Axial view of the T2-weighted image showing the cartilaginous cap at the rightmost extension site (arrow). The left hypoglossal nerve (arrowhead) is visualized; meanwhile, the right hypoglossal nerve is not visualized because of its engulfment by the tumor. The medulla oblongata and basilar artery are compressed by the tumor. D and E: Axial views of the gadolinium-enhanced T1weighted image with fat suppression revealing the tumor extending into the right JF and compressing the right jugular bulb (arrowhead). Only the lateral margin of the cartilaginous cap is enhanced (arrows). 
clivus, which was accompanied by a cartilaginous cap in the right lateral part adjacent to the jugular foramen (JF). Only the lateral margin of the cartilaginous cap was intensified on gadolinium-enhanced T1weighted imaging. The tumor extended to the bilateral hypoglossal canal (HGC) and JF, both of which were engulfed by the tumor on the right side, whereas the medulla oblongata and right vertebral artery were compressed at the craniocervical junction. Compared with findings on MRI performed 8 years earlier, the cartilaginous cap adjacent to the JF had clearly increased in size. Physical findings included hoarseness, dysphagia, tongue deviation to the right side, and marked atrophy of the right side of the tongue and right sternocleidomastoid muscle. Otolaryngological fiberscopic observation revealed salivary retention in the pyriform fossa and diminished right vocal cord movement, suggesting right recurrent laryngeal nerve palsy. These findings informed a diagnosis of right lower cranial nerve $(C N I X, X$, and XI) and hypoglossal nerve (CN XII) palsies. EES was indicated to prevent the contralateral lower CN palsy, decompress the brainstem, and pathologically determine tumor malignancy.

\section{Surgical Procedure}

We performed EES via the bilateral transmaxillary-pterygoid and translacerum approach ${ }^{8}$ (extreme far-medial approach) using neuronavigation, neuromonitoring of the transcranial motor evoked potentials, and a nerve integrity monitor (NIM-Response, Medtronic Xomed) for CNs IX, XI, and XII. A nasoseptal flap was prepared on the left side for dural reconstruction before the bilateral medial maxillectomy with preservation of the nasolacrimal ducts, and wide sphenoidectomy was performed to secure the working space. After the orbital process of the palatine bone was resected, the sphenopalatine artery was coagulated on the right side. Then, the posterior wall of the maxillary sinus was drilled out to expose the pterygopalatine fossa. The bilateral vidian nerves, descending palatine arteries, and greater palatine nerves were preserved. The contents of the pterygopalatine fossa were subperiosteally dissected from the pterygoid plate, which was then drilled out. The nasopharyngeal mucosa was incised in an inverted $U$ shape, carefully preserving the eustachian tubes, before the mucosa with the prevertebral muscle and ligaments were caudally reflected to expose the upper edge of the anterior arch of the atlas. Using neuronavigation, the bone was drilled from the inferior wall of the sphenoid sinus to the lower clivus and the anteromedial side of the bilateral HGCs while the course of the internal carotid artery (ICA) was confirmed with Doppler ultrasound. The fibrocartilaginous tissue around the foramen lacerum was cut just beneath the vidian nerve to allow inferior mobilization of the eustachian tube (translacerum approach ${ }^{8}$ ) and facilitate bony tumor resection in the jugular tubercle and occipital condyle. The fibrous bone tissue of the tumor was drilled under observation with a $30^{\circ}$ endoscope.

After resection of the fibrous bone tissue, a cartilaginous cap layer was found between the fibrous bone tissue and posterior fossa dura mater. Because this cap did not adhere to the dura mater, it was relatively easy to separate. The lower clivus dura was ventrally bulged by cerebrospinal fluid (CSF) pressure, confirming successful decompression of the brainstem. A small, weeping CSF leak was found in the thin area of the clival dura and primarily covered with fibrin glue-soaked Gelfoam (Pfizer). A $70^{\circ}$ endoscope was also used to resect the most laterally located tumor just inferior to the petrous ICA and medial to the ICA at the carotid foramen on the right side. Extensive cartilaginous cap tissue was found there and carefully resected. After the complete resection in this area, the ICA around the carotid foramen was exposed. The bilateral lower $\mathrm{CNs}$ in the dura mater around the JF were preserved while their courses were confirmed with the NIM-Response. The left CN XII was localized using the NIM-Response and preserved, but the right CN XII could not be localized because of its preoperative complete palsy. After filling the dead space of the resection cavity with abdominal fat tissue on the right side, the clivus dura was covered with a nasoseptal flap. The inverted U-shaped nasopharyngeal mucosal flap was repositioned and fixed with fibrin glue before gentle buttressing with Sorbsan dressing (Alcare) (Video 1).

VIDEO 1. Clip showing bilateral transmaxillary-pterygoid and extreme far-medial endoscopic endonasal approach for an extensive lower clivus osteochondroma in a patient with HME. Click here to view.

\section{Postoperative Course}

CT (Fig. 3) on postoperative day 1 and MRI (Fig. 4) on postoperative day 7 confirmed a nearly total resection of the tumor and sufficient volume reduction in line with the preoperative goals of preserving the lower $\mathrm{CN}$ and decompressing the brainstem. Because the patient suffered from paresis of the right lower $\mathrm{CN}$ and $\mathrm{CN}$ XII preoperatively, the bone lesion adjacent to the left lower $\mathrm{CN}$ was intentionally preserved to avoid postoperative bilateral paresis of lower CN or CN XII (Fig. 4D), considering the patient's quality of life as the first priority. However, we observed transient worsening of dysphagia. Otolaryngological fiberscopy revealed intact left $\mathrm{CN}$ $\mathrm{IX}, \mathrm{X}$, and $\mathrm{XII}$ function and unchanged right $\mathrm{CN} X$ but slightly worsened right CN IX function. In addition, the entire pharynx was swollen. Therefore, the patient was temporarily fed via a nasogastric tube, and a swallow training diet was started on postoperative day 5. This tube was removed on postoperative day 18 after the symptoms resolved. No other complications, including CSF leakage or infections, were encountered, and the nasal packing was removed 6 days after EES. We made the pathological diagnosis of osteochondroma (exostosis) (Fig. 5) because no malignancy was found, even in the cartilaginous cap tissue. The patient was discharged after $\mathrm{CN}$ $\mathrm{IX}, \mathrm{X}, \mathrm{XI}$, and XII symptoms improved to the preoperative level. Because cervical spine CT 1 month after surgery demonstrated instability and local lateral tilt in the right atlanto-occipital joint accompanying cervical scoliosis, posterior occipitoaxial fixation was performed 3 months after EES. Otherwise, the postoperative course was uneventful, and the patient remains under outpatient follow-up. After the 4-month follow-up visit, he attained a Karnofsky Performance Scale score of 80 .

\section{Discussion \\ Observations}

$\mathrm{HME}^{1-6}$ is a disease in which benign tumors (exostoses or osteochondromas) frequently develop in bones throughout the body along with subcutaneous masses. Tumors often appear bilaterally and frequently in the distal femur and proximal tibia around the knee. Most exostoses have a metaphyseal mass that impairs the mobility of the surrounding joint, stunting epiphyseal growth, causing abnormal alignment, and, in severe cases, inducing dysfunction of the upper and lower limbs. ${ }^{1,2}$ HME is a genetically heterogeneous autosomal dominant disorder, ${ }^{1-6}$ and three HME 

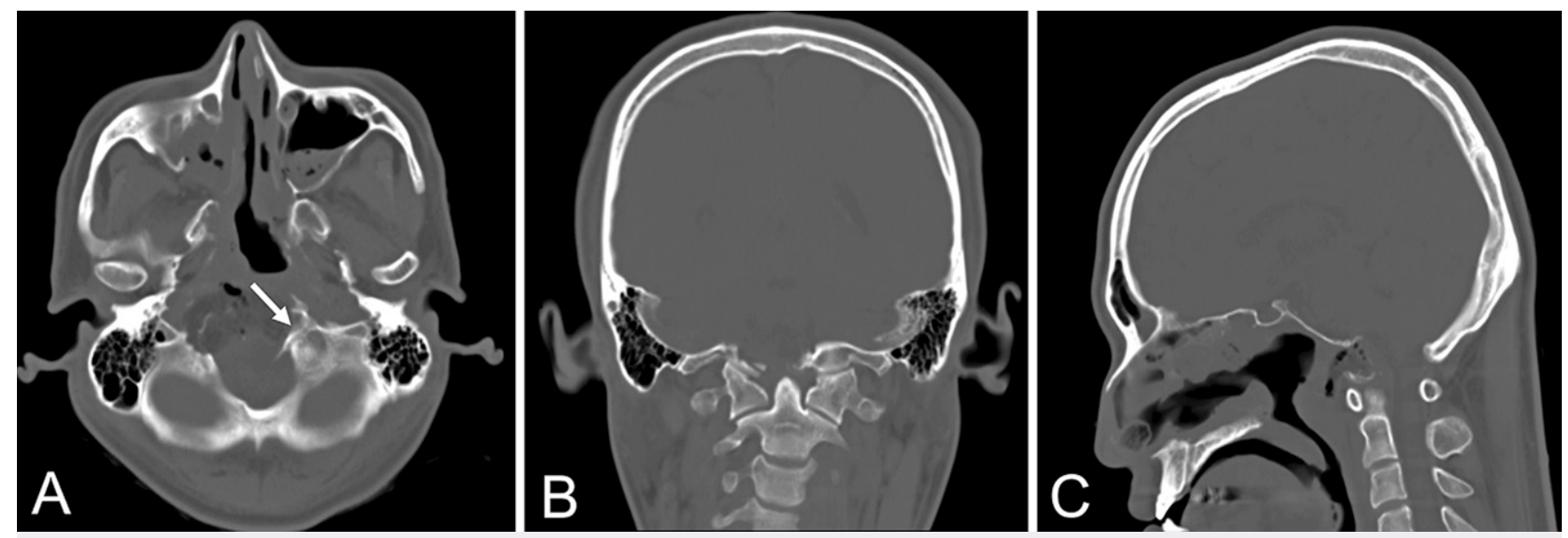

FIG. 3. Postoperative CT. Axial (A), coronal (B), and sagittal (C) views showing nearly complete resection of the tumor. The cortex of the left HGC (arrow) is preserved $(\mathbf{A})$.

loci have been mapped to chromosomes 8q24 (EXT1), 11p11-13 (EXT2), and 19p (EXT3). ${ }^{7}$ Although the precise incidence of HME in Japan is unclear, it is reported to be 1 in 50,000 among White persons. $^{9}$

The epiphyseal line formed after puberty stops the growth of exostoses, but malignancy should be evaluated when the thickness of the cartilaginous cap exceeds $2 \mathrm{~cm}$ or if its mass increases rapidly in adulthood. Although chondrosarcoma accounts for most of these
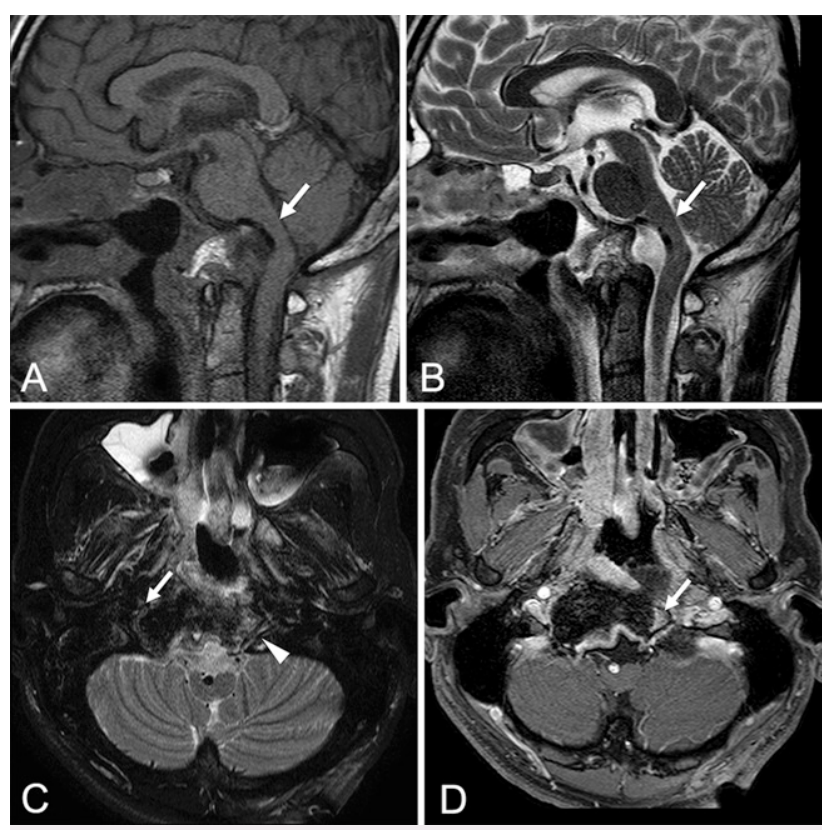

FIG. 4. Postoperative MRI. A and B: Sagittal views of the T1- and T2-weighted images reveal successful decompression of the brainstem (arrows). C and D: Axial views of the T2-weighted and gadolinium-enhanced T1-weighted image with fat suppression showing nearly complete removal of the tumor and disappearance of the cartilaginous cap at the rightmost extension site (C, arrow). The left hypoglossal nerve (arrowhead) is preserved. A small amount of residual tumor is found in the left occipital condyle (D, arrow). cases, they may also develop into osteosarcomas or fibrosarcomas, with a reported incidence of approximately $5 \% .{ }^{1-6}$ In the case involving our patient, although the possibility of a malignant tumor (especially for chondrosarcoma) was preoperatively considered because of the symptomatic clivus lesion with a growing cartilaginous cap, pathology discovered exostosis and ruled out malignant transformation. Although the cartilaginous cap was totally resected, vigilant future follow-ups are necessary in our patient.

Treatment is commonly provided for symptomatic cases and those in which malignant transformation is possible ${ }^{1-6}$ because a complete en bloc resection of the tumor (especially of the cartilaginous cap) prevents recurrence. ${ }^{1,10}$ Some studies have shown that HME can develop exostoses at the skull base and that osteochondroma formation is amenable to bone morphogenetic protein (BMP) signaling antagonist intervention. ${ }^{3}$ In vivo research has demonstrated that systemic

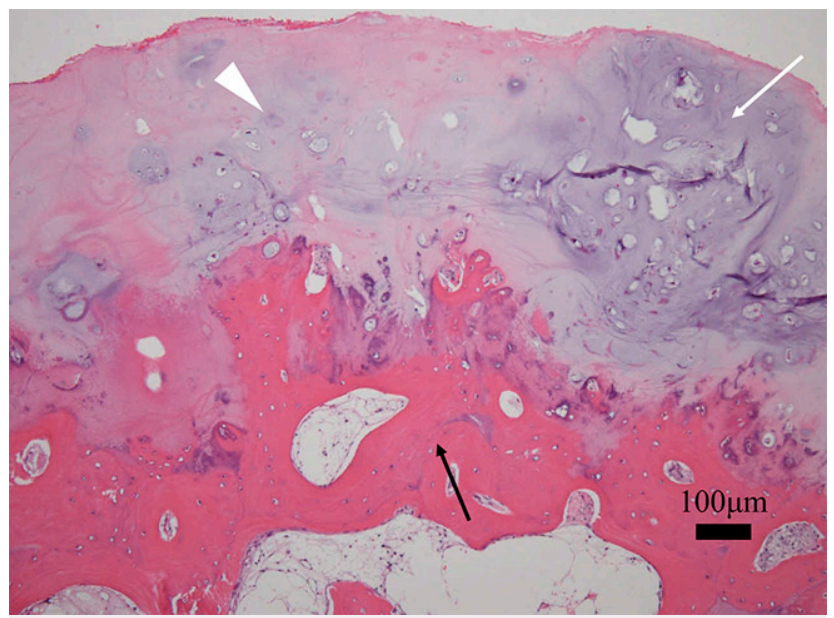

FIG. 5. Hematoxylin and eosin stain shows pathological findings. Purple (cartilage, white arrow) and pink (bone, black arrow) components, plus partially gray areas outside the pink areas, are typical for a cartilaginous cap (arrowhead). The chondrocyte component is enriched with cellular components but lacks nuclear atypicality, which points to osteochondroma and away from chondrosarcoma. Bar $=100 \mu \mathrm{m}$. 
administration of selective BMP inhibitor LDN-193189 effectively inhibits osteochondroma growth in conditional EXT1-mutant mice. ${ }^{3}$ However, the treatment of extensive lower clivus lesions, as in our patient, remains challenging in the face of solid, bony mass lesions that compress the brainstem because of the risk of brainstem damage, lower $\mathrm{CN}$ dysfunction, or postoperative CSF leakage due to extensive clival dural defects. In these cases, using EES for clivus lesion extraction protects against brain damage, especially when the extreme far-medial approach, ${ }^{11,12}$ including the transmaxillary-pterygoid and translacerum approaches, ${ }^{8}$ is adopted because it secures a sufficient working space. Although the potentially malignant cartilaginous cap must be localized by drilling deeply into the solid bony tissue (because of the presence of the cartilaginous cap layer between the solid bone and dura mater), the cap tissue can be safely resected and easily dissected from the dura mater without creating large dural defects as we experienced in this patient.

To the best of our knowledge, this is the first case in which EES was performed to address a clivus osteochondroma in a patient with HME. Although reports of clivus lesions developing from HME are extremely rare, Sinha et al. ${ }^{3}$ reported that nearly half of the $50 \mathrm{HME}$ patients in their study exhibited moderate defects or osteochondroma-like outgrowths in the clivus. However, detailed surgical cases have not been reported for skull base types of exostosis, including clivus lesions in patients with HME. In case of a recurrence in our patient, we are considering a reoperation using the same approach, and we hope that the aforementioned systemic administration of selective BMP inhibitor will become an alternative treatment option in the future.

\section{Lessons}

Clivus lesions in patients with HME are extremely rare, and surgical treatment involving pathological diagnosis is recommended for symptomatic lesions and patients at risk of malignant transformation (e.g., chondrosarcoma, osteosarcoma, or fibrosarcoma), especially in cases associated with a growing cartilaginous cap. Because EES creates a wide working space, using a transmaxillary-pterygoid or extreme farmedial approach offers maximum safe resection and an optimistic prognosis, even in the complex case we encountered. Although a single case experience may not be generalized, we hope that this finding is helpful for surgeons who treat such challenging conditions and that a comprehensive study of clivus lesions in HME will aid in establishing evidence-based therapies with favorable long-term clinical outcomes.

\section{Acknowledgments}

We thank Bryan J. Mathis (Medical English Communication Center, University of Tsukuba) for language revision of the manuscript and English audio narration of the video. Moreover, we thank Dr. Alexander Zaboronok, Department of Neurosurgery, for help with the language editing of the manuscript.

\section{References}

1. Jurik AG. Multiple hereditary exostoses and enchondromatosis. Best Pract Res Clin Rheumatol. 2020;34(3):101505.

2. D'Arienzo A, Andreani L, Sacchetti F, et al. Hereditary multiple exostoses: current insights. Orthop Res Rev. 2019;11:199-211.
3. Sinha S, Mundy C, Bechtold T, et al. Unsuspected osteochondroma-like outgrowths in the cranial base of hereditary multiple exostoses patients and modeling and treatment with a BMP antagonist in mice. PLoS Genet. 2017;13(4):e1006742.

4. Ruiz LP, Lara JC. Craniomaxillofacial features in hereditary multiple exostosis. J Craniofac Surg. 2012;23(4):e336-e338.

5. Liang C, Wang YJ, Wei YX, et al. Identification of novel EXT mutations in patients with hereditary multiple exostoses using wholeexome sequencing. Orthop Surg. 2020;12(3):990-996.

6. Jurik AG, Jørgensen PH, Mortensen MM. Whole-body MRI in assessing malignant transformation in multiple hereditary exostoses and enchondromatosis: audit results and literature review. Skeletal Radiol. 2020;49(1):115-124.

7. Francannet $\mathrm{C}$, Cohen-Tanugi A, Le Merrer M, et al. Genotype-phenotype correlation in hereditary multiple exostoses. J Med Genet. 2001;38(7):430-434.

8. Taniguchi M, Akutsu N, Mizukawa K, et al. Endoscopic endonasal translacerum approach to the inferior petrous apex. J Neurosurg. 2016;124(4):1032-1038.

9. Signori E, Massi E, Matera MG, et al. A combined analytical approach reveals novel EXT1/2 gene mutations in a large cohort of Italian multiple osteochondromas patients. Genes Chromosomes Cancer. 2007:46(5):470-477.

10. Asari T, Echigoya N, Sasaki N, et al. Cervical myelopathy due to a solitary osteochondroma: a case report. Springerplus. 2016; 5:535.

11. Silveira-Bertazzo G, Manjila S, London NR Jr, Prevedello DM. Techniques and challenges of the expanded endoscopic endonasal access to the ventrolateral skull base during the "far-medial" and "extreme medial" approaches. Acta Neurochir (Wien). 2020;162(3): 597-603.

12. Vaz-Guimaraes F, Nakassa ACl, Gardner PA, et al. Endoscopic endonasal approach to the ventral jugular foramen: anatomical basis technical considerations, and clinical series. Oper Neurosurg (Hagerstown). 2017;13(4):482-491.

\section{Disclosures}

The authors report no conflict of interest concerning the materials or methods used in this study or the findings specified in this paper.

\section{Author Contributions}

Conception and design: Akutsu, Morinaga. Acquisition of data: Akutsu, Morinaga. Analysis and interpretation of data: Akutsu, Morinaga. Drafting the article: Akutsu, Morinaga. Critically revising the article: all authors. Reviewed submitted version of manuscript: all authors. Approved the final version of the manuscript on behalf of all authors: Akutsu. Statistical analysis: Morinaga. Administrative/technical/material support: Morinaga, Kino, Yasuda. Study supervision: Akutsu.

\section{Supplemental Information \\ Videos}

Video 1. https://vimeo.com/515337091.

\section{Correspondence}

Hiroyoshi Akutsu: University of Tsukuba, Tsukuba, Ibaraki, Japan. akutsuh@md.tsukuba.ac.jp. 